\title{
The Increased Production Efficiency and Optimization Terms of Stationarity by Flat Grinding with Abrasive Circle Surface
}

\author{
Husseinov Hassan Ahmad, ${ }^{1}$ Bagirov Sahib Abbas, ${ }^{1}$ Radoslav Krehel', ${ }^{2}$ and Marek Kočiško ${ }^{2}$ \\ ${ }^{1}$ Technological Systems and Special Equipment, Azerbaijan Technical University, H. Cavid Avenue, Baku AZE1073, Azerbaijan \\ ${ }^{2}$ Department of Computer Aided Manufacturing Technologies, Faculty of Manufacturing Technologies with a Seat in Prešov, \\ Technical University of Košice, Štúrova 31, 08001 Prešov, Slovakia
}

Correspondence should be addressed to Radoslav Krehel'; radoslav.krehel@gmail.com

Received 27 August 2014; Revised 27 October 2014; Accepted 19 November 2014; Published 11 December 2014

Academic Editor: Rui Vilar

Copyright (c) 2014 Husseinov Hassan Ahmad et al. This is an open access article distributed under the Creative Commons Attribution License, which permits unrestricted use, distribution, and reproduction in any medium, provided the original work is properly cited.

\begin{abstract}
This scientific work deals with the production area. The paper investigates the problems related to the uneven abrasive effect on the processed surface by flat grinding with the abrasive circle surface, and the analytical expression of pattern distribution of the working abrasive grain within the limits of various technological primitives and inaccuracy of geometric shape have been determined. The ways of stationary provision of the grinding surface have been suggested. This paper also focuses on the economic efficiency of the production.
\end{abstract}

\section{Introduction}

One of the most widespread methods of the final process of the flat surface detail is considered to be the flat grinding with the abrasive circle surface. High quality of the surface by face grinding is provided for the productivity or low prime costs.

Micro- and macro-offset forming mechanisms by flat grinding with the abrasive circle surface have their own specific peculiarities. Grain situated on the operating circle by different circumferential speed is supposed to be one of them. Speed variation of the grain situated on the same circle takes place on different circles due to the direction and dimension. Speed variation of the cutting of abrasive grains along with the contact area occurs according to two arguments, that is, from the angle of rotation $\varphi$ and radius $R$ and position of the grain with respect to the circle centre. Consequently, cutting speed of every practically operating grain in the contact area is considered to be the varying vector, that is, vector function of two scalar variables $\varphi$ and $R$, as every value of $\varphi$ and $R$ within the contact area coincides with a certain value of the cutting speed. Change of speed of cutting of the grains located on one circle in direct ratio to width of the ground surface, detail speed, and in inverse proportion to diameter circle [1].

\section{Description of the Issue}

A grinding wheel is a self-sharpening tool composed of discrete abrasive grains held together by a bonding agent with composite structure of clearance allowance for the cutting edges. The characteristics of a grinding wheel depend on the combined elements of the abrasive, grit size, grade, structure, and bond (Figure 1). follows.

The main components of the grinding wheel are as

Abrasive. The abrasive grain is the element that actually performs the cutting activity in the grinding process. And the choice of the abrasive grain depends on the material to be ground.

Bond. The role of bond is to hold the individual grains together. The type of the bond depends on the operating speed of the wheel, the type of operation, and the surface finish required.

Pore. Pore exists between the grains and the bond. In order to provide the chip clearance, the air space (pore) must exist 


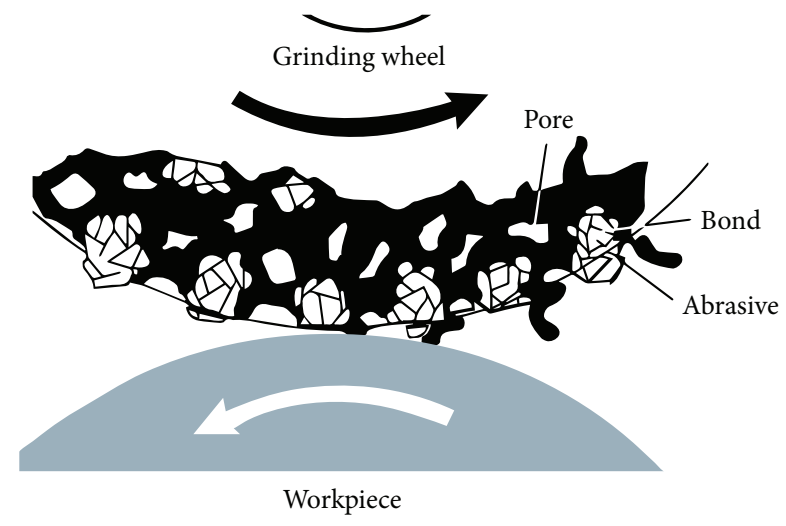

FIGURE 1: Basic principles of grinding.

between the grains and the bond. Dense spacing is denoted by low numbers and open spacing by high numbers $[2,3]$.

The relative holding power of the bond which holds the abrasive grains within a wheel-degree of "hardness" of strength is indicated by softer grades in the low alphabet and harder grades in the high alphabet.

Figure 2 shows abrasive grains with light bond coating and weak connecting bond post as in a relatively soft grade wheel. (Bright areas are the pores required for chip clearance.)

Figure 3 shows abrasive grains of the same size but with heavier bond coating and thicker and stronger bond posts as in a hard grade wheel.

Figure 4 shows normal grinding and abnormal grinding.

Wide range of the stochastic phenomena occurring in the course of face grinding besides high efficiency this process causes also numerous shortcomings, the main of which are: large heat build-up, unfavorable conditions of the swarf and heat removal, for feeding the lubricating-cooling fluid in the cutting area, uneven abrasive effect on the machined surface, and so forth. All these disadvantages in total have negative influence on forming the geometrical parameters of surfaces and mechanical and physical peculiarities of the surface layer [4-7].

Flat grinding with the abrasive circle differs with more contact area and hence with more extension of the technological primitives of the input and output. Thus unlike peripheral grinding technological primitives are formed on length of the working course in inverse proportion to diameter circle. Breakdown of stationary grinding surface has its site along the processing length, in and against the feed of the detail in the abrasive grain operating areas, concerning the reference axis of the grinding wheel in the direction of machine table movement, and along the processing within the technological primitives of the input and output and alteration of the configuration of the grinding surface $[6,8]$.

For the identification of the physical nature of the processes through the processing of the width face grinding is to be analyzed at symmetrical position of the workpiece. Symmetry axis of the sphere divides the cutting zone into two parts. Depending on the feed direction of the piece grain in one part works against the feed and in feed direction

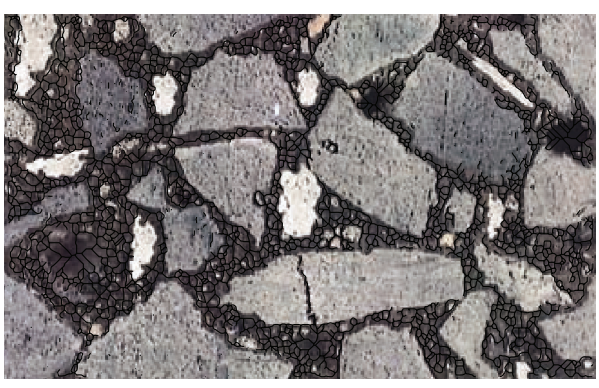

Figure 2: Composition A.

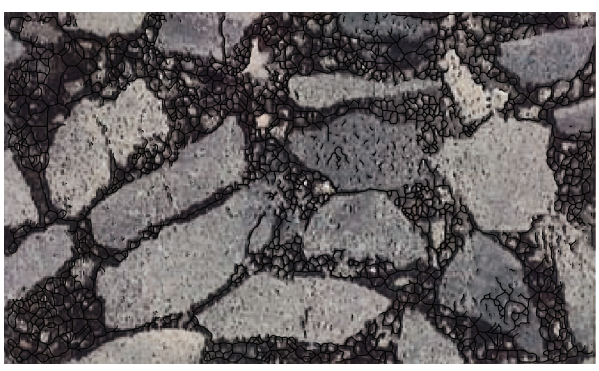

Figure 3: Composition B.

on the other part. In working against the feed direction the grain undergoes high loads whereby the speed of cutting changes from minimum to average. Violation stationarities of a polished surface takes place on processing width, in zones of work of abrasive grain on and against giving of a detail, concerning an axis symmetry of a grinding wheel in the direction of the speed of the movement of a table of the machine and on processing length within technological primitives of an entrance and an exit and changes of a configuration of the ground surface.

\section{Theoretical Assumptions}

At face grinding, the peripheral conventional stripes (lines) with uneven abrasive effect have the forms of concentric circle areas with the width of equal longitudinal feed for one revolution of the disk (Figure 5).

To determine the distribution pattern of the quality of practically operating grains over the working surface of face grinding the following steps are supposed.

The area of the working surface disk is

$$
F_{T}=\Pi b D_{c} .
$$

Area $j$ conventional line is determined according to the following formula:

$$
F_{j}=\Pi S_{l} D_{c j}
$$

Longitudinal feed per revolution of the disk is

$$
S_{l}=\frac{V_{d} \pi D_{k}}{1000 V_{k}} .
$$




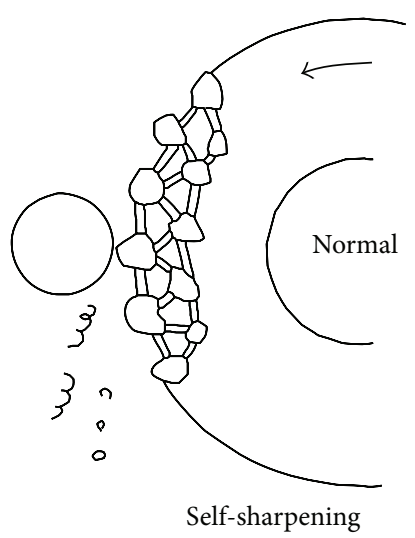

(a)

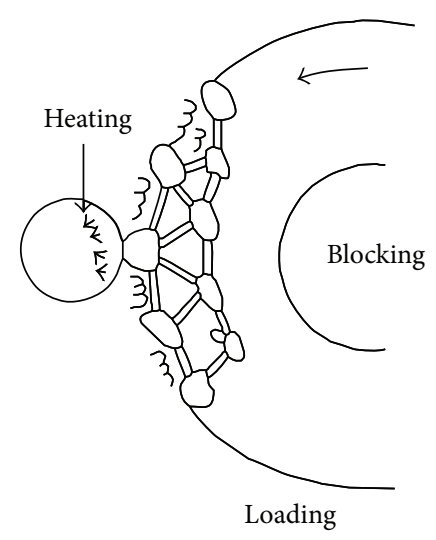

(b)

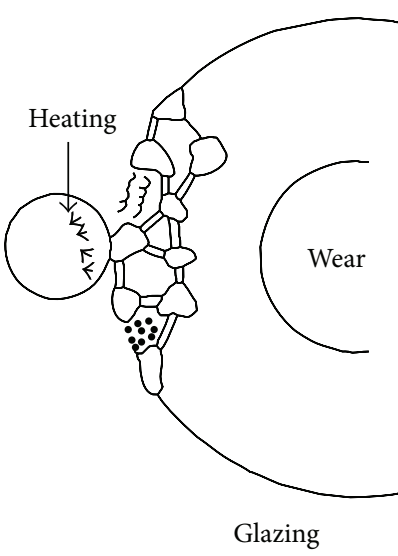

(c)

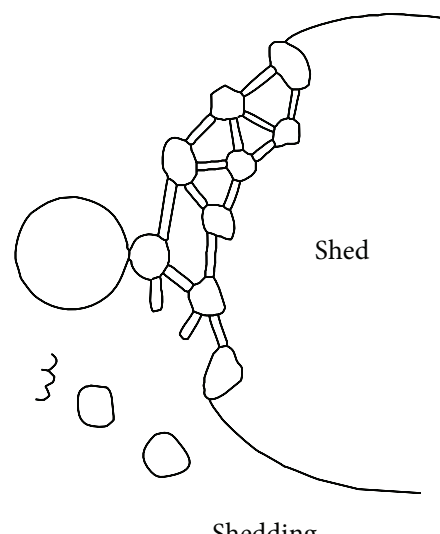

(d)

Figure 4: Phase grinding.

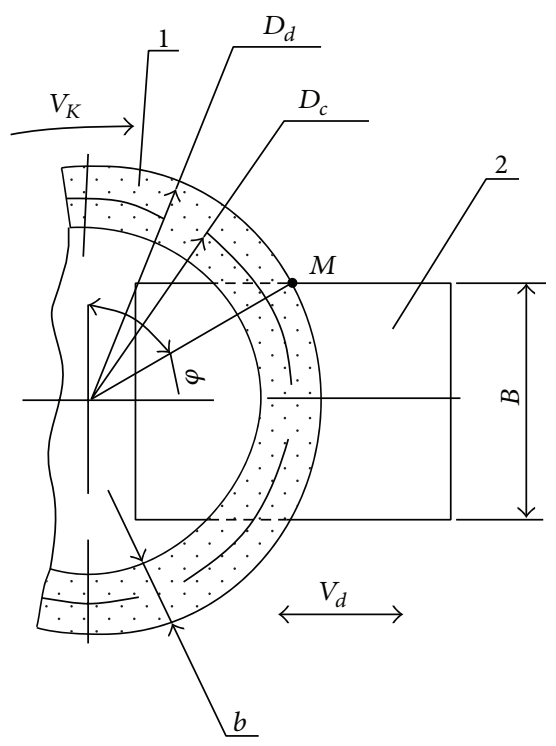

FIGURE 5: Diagram of flat grinding with abrasive circle surface.

The pitch diameter of the current conventional line is considered to be expressed through sheer parameters of the grinding process:

$$
D_{c j}=D_{k}-(2 j-1) S_{l}
$$

Thereby,

$$
F_{j}=\frac{\pi^{2} V_{d} D_{k}}{1000 V_{k}}\left[D_{k}-(2 j-1) \frac{\pi^{2} V_{d} D_{k}}{1000 V_{k}}\right] .
$$

The number of practically operating grains on the working surface wheel taking into account the hit percentage into cut areas may be shown in further contact as follows:

$$
0<j \leq \frac{b}{S_{l}} .
$$

The value of $F_{1}$ is supposed to be made out of formula (5) into formula (6), using some simplifications:

$$
\begin{aligned}
& i_{f}=i \pi S_{l}[\left(D_{d}+D_{d} q+D_{d} q^{2}+\cdots+D_{d} q^{j-1}\right) \\
&\left.-\left(S_{l}+3 S_{l} q+5 S_{l} q^{2}+\cdots+(2 j-1) S_{l} q^{j-1}\right)\right] \\
& 0<j \leq \frac{b}{S_{l}} .
\end{aligned}
$$

As it is seen, the first part of the formula (7) presents its geometrical progression, and the formula of the amount of practically working grains on the circle surface will be

$$
\begin{array}{r}
i_{f}=i \pi S_{l}\left[D_{d} \frac{\left(1-q^{j}\right)}{1-q}-\sum_{1}^{j}(2 j-1) S_{l} q^{j-1}\right] \\
0<j \leq \frac{b}{S_{l}} .
\end{array}
$$

Number of practically working grains on the circle surface of the grinding wheel using the formula of active grains amount per $1 \mathrm{~mm}^{2}$ of wheel surface will be presented as follows: [9]

$$
\begin{aligned}
& i_{f}= \frac{0,167 \beta \sqrt{k}}{\alpha^{3 / 4} \sqrt{\operatorname{tg} \gamma \bar{X}_{j}^{2} \sqrt{1-\varepsilon}}} \sqrt{\frac{\omega}{1000 V_{k}}} \pi S_{l} \\
& \times\left[\frac{D_{d}\left(1-q^{j}\right)}{1-q}-\sum_{1}^{j}(2 j-1) S_{l} q^{j-1}\right] \\
& 0<j \leq \frac{b}{S_{l}} .
\end{aligned}
$$

Amount of actually working abrasive grains within the area contact we will define by multiplication of amount of actually working grains at a working end face of a grinding wheel on a ratio of length of an arch of contact the grains 
which are on average diameter on circle length also on to average diameter of an end face of a grinding wheel; that is,

$$
i_{k}=i_{f} \frac{L_{k_{c}}}{\pi D_{c}} .
$$

The pattern of the change amount of the practically operating abrasive grains within the contact area of the cup grinding wheel with machined surface [10], given analytical expression of the contact arc, will be presented as follows: for technological primitive input,

$$
\begin{aligned}
i_{k_{2}}= & \frac{0,167 \beta \sqrt{k}}{\alpha^{3 / 4} \sqrt{\operatorname{tg} \gamma} \bar{X}_{j}^{2} \sqrt{1-\varepsilon}} \sqrt{\frac{\omega}{1000 V_{k}}} S_{l} \frac{\alpha \sqrt{1+\left(V_{d}^{2} /\left(60 V_{k c}\right)^{2}\right)}}{2} \\
& \times\left[\frac{D_{d}\left(1-q^{j}\right)}{1-q}-\sum_{1}^{j}(2 j-1) S_{l} q^{j-1}\right], \quad 0<j \leq \frac{b}{S_{l}} .
\end{aligned}
$$

For the technological primitive set grinding,

$$
\begin{aligned}
i_{k_{y c m}}= & \frac{0,167 \beta \sqrt{k}}{\alpha^{3 / 4} \sqrt{\operatorname{tg} \gamma} \bar{X}_{j}^{2} \sqrt{1-\varepsilon}} \\
& \times \sqrt{\frac{\omega}{1000 V_{k}} S_{l} \frac{\alpha \sqrt{1+\left(V_{d}^{2} /\left(60 V_{k c}\right)^{2}\right)}}{2}} \\
& \times\left[\frac{D_{d}\left(1-q^{j}\right)}{1-q}-\sum_{j=i}^{b / S_{l}}(2 j-1) S_{l} q^{j-i}\right], \quad 0<j \leq \frac{b}{S_{l}} .
\end{aligned}
$$

For a technological primitive of an entrance.

The determined patterns of the uneven abrasive impact at flat grinding with abrasive circle surface indicate the unevenness of the spring pressing of the technological structure along the length of the machining step. Due to the significance of the consistent patterns character of spreading the uneven abrasive impact, machining step was conditionally divided into technological primitives of input, stationary grinding, offset, and output. Consequently, the machine of the shape inaccuracy formation by face grinding has characteristic features within the limits of abovementioned technological primitives. Let us analyze each of them separately [11-13].

Let us consider the cutting force at grinding as the sum of the microcutting power of separate single-piece grains in the contact area. Let the microcutting power value within the limits of contact area be stable and equal to the arithmetic mean value of $\rho_{c p}$. Consider

$$
P_{y}=\rho_{c p} i_{k}
$$

A specific fault of the shape occurs by the grinding of even surfaces with sphere face: concavities and bulbs. The physical nature of the shape faults forming process can be explained by the changing cutting force through the length of the pass due to changes in the current contact surface. In the beginning of the grinding, the contact surface is close to zero, so the cutting force and flexible deformations are practically absent. In a technological primitive of an entrance, the contact surface increases up to the established value and cutting force and flexible deformations increase up to the value corresponding to the established process. A reverse case occurs in the process output primitive; that is, the contact surface and subsequently the cutting force and flexible deformations decrease from the value corresponding to the process primitive of the established grinding to zero. Distortion of the levelness of grinded surface in longitudinal section occurs as a result of these cases.

At stable rigidity of technological system on processing length unevenness of elastic otzhatiya occurs because of change of a normal component of force of cutting. Within a technological primitive of an entrance unevenness of a normal component of force of cutting is defined by a difference of its values corresponding and $j=X_{i} / S_{l}=1$ and $j=b / S_{l}$. Let us determine the smallest and largest value of the regular making-up of the cutting force in the technological primitive of the input. Consider

$$
\begin{aligned}
& P_{y_{B x_{M u H}}=} \frac{0,167 \beta \sqrt{k}}{\alpha^{3 / 4} \sqrt{\operatorname{tg} \gamma} \bar{X}_{j}^{2} \sqrt{1-\varepsilon}} \sqrt{\frac{\omega}{1000 V_{k}}} S_{l} \rho_{c p} \\
& \times \frac{\alpha \sqrt{1+\left(V_{d}^{2} /\left(60 V_{k c p}\right)^{2}\right)}}{2}\left(D_{k}-S_{l}\right) \quad j=1 \\
& P_{y_{\text {Max }}=} \frac{0,167 \beta \sqrt{k}}{\alpha^{3 / 4} \sqrt{\operatorname{tg} \bar{X}_{j}^{2} \sqrt{1-\varepsilon}}} \\
& \times \sqrt{\frac{\omega}{1000 V_{k}} S_{l} \rho_{c p} \frac{\alpha}{1+\left(V_{d}^{2} /\left(60 V_{k_{c p}}\right)^{2}\right)}} \\
& \times\left[\frac{D_{k}\left(1-q^{b / S_{l}}\right)}{1-q}-\left(\frac{2 b}{S_{l}}-1\right) S_{l} q^{\left(b / S_{l}\right)-1}\right], \\
& j=\frac{b}{S_{l}} .
\end{aligned}
$$

The unevenness of the cutting force causes the unevenness of the spring pressing of the technological structure and this leads to the formation of the shape inaccuracy:

$$
\Delta_{\Phi}=\frac{P_{y_{\max }}-P_{y_{B x_{M u H}}}}{J_{c p}}=\frac{\Delta P_{y}}{J_{c p}} .
$$

Recording the values $P_{y_{B x M U H}}$ and $P_{y_{y c m}}$, respectively, from (14) and (15) to the (16), we will get an analytical expression 
of the shape inaccuracy in the technological primitive of the input. Consider

$$
\begin{aligned}
& \Delta_{\phi_{B b l x_{\text {Max }}}} \\
& =\frac{0,167 \beta \sqrt{k}}{\alpha^{3 / 4} \sqrt{\operatorname{tg\gamma } \bar{X}_{j}^{2} \sqrt{1-\varepsilon}}} \\
& \quad \times \sqrt{\frac{\omega}{1000 V_{k}} \frac{\rho_{c p} S_{l} \alpha \sqrt{1+\left(V_{d}^{2} /\left(60 V_{k}\right)^{2}\right)}}{2 J_{c p}}} \\
& \quad \times\left[\frac{D_{k} q\left(1-q^{\left(b / S_{l}\right)-1}\right)}{1-q}+S_{l}-\sum_{1}^{b / S_{n p}}(2 j-1) S_{l} q^{j-1}\right], \\
&
\end{aligned}
$$

The differential of the cutting force in the technological primitive of the output contains

$$
\Delta P_{y}=P_{y_{e c n}}-P_{y_{B b l x M u H}} .
$$

The smallest value of the making-up of the cutting force in the technological primitive of the output coincides with the last conditional line with the width $S_{l}$ of the operating face circle. Consider

$$
\begin{aligned}
P_{y_{B b l \times M u}}= & \frac{i S_{l} \rho_{c p} \alpha \sqrt{1+\left(V_{d}^{2} /\left(60 V_{k c p}\right)^{2}\right)}}{2} q^{\left(b / S_{l}\right)-1} \\
& \times\left[D_{k}-\left(2 \frac{b}{S_{n \mathrm{p}}}-1\right) S_{l}\right] .
\end{aligned}
$$

Accordingly, the unevenness of the cutting force is determined as the diminution of forces between the technological primitive of the stationary grinding (15) and the output (19). Consider

$$
\begin{aligned}
P_{y_{\text {Max }}}= & \frac{i S_{l} \rho_{c p} \alpha \sqrt{1+\left(V_{d}^{2} /\left(60 V_{k_{c p}}\right)^{2}\right)}}{2} \\
& \times\left[\frac{D_{k}\left(1-q^{b / S_{l}}\right)}{1-q}-\sum_{1}^{b / S_{l}}(2 j-1) S_{l} q^{j-1}\right] .
\end{aligned}
$$

The analysis of the given formulae shows that largest value of the unevenness of the regular making-up of the cutting force, in the absence of the offset on the grinding surface, coincides with the technological primitives of the output. Analytical expressions of the shape inaccuracy in the

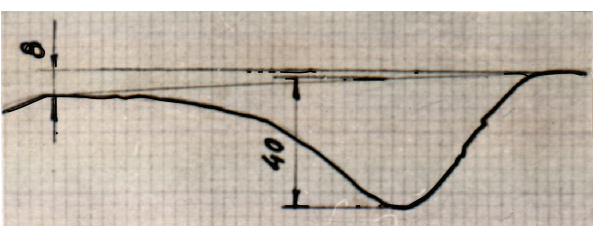

(a)

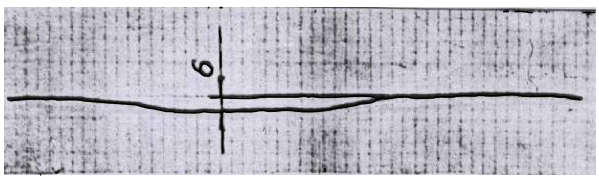

(b)

FIGURE 6: Profile diagram of unstraightness along the length of processing, vertical magnification $\times 500$, and horizontal magnification $\times 0,5$ : (a) without the use of the program device and (b) with the use of the program device.

technological primitive of the output will be presented as follows:

$$
\begin{aligned}
& \Delta_{\phi_{B b l x_{\text {Max }}}=} \frac{0,167 \beta \sqrt{k}}{\alpha^{3 / 4} \sqrt{\operatorname{tg} \gamma} \bar{X}_{j}^{2} \sqrt{1-\varepsilon}} \\
& \times \sqrt{\frac{\omega}{1000 V_{k}} \frac{\rho_{c p} S_{l} \alpha \sqrt{1+\left(V_{d}^{2} /\left(60 V_{k}\right)^{2}\right)}}{2 J_{c p}}} \\
& \times\left[\frac{D_{k}\left(1-q^{\left(b / S_{l}\right)-1}\right)}{1-q}-\sum_{1}^{\left(b / S_{l}\right)-1}(2 j-1) S_{l} q^{j-2}\right], \\
& 0<j \leq \frac{b}{S_{l}}-1 .
\end{aligned}
$$

The analysis has shown that the shape inaccuracy value of the technological primitive of the input is approximately $10 \%$ more than in the technological primitive of the output. The inaccuracy feature is identical to the spreading feature of the uneven abrasive impact on the machined surface. The value and inaccuracy feature are influenced by the dimension and characteristics of the grinding circle, elements of cutting conditions, stiffness of technological structure, and peculiarities of the flat grinding with the abrasive circle surface process. The length of the technological primitives of the input and output and thereby the reach areas of the shape inaccuracy along the length of the processing are determined by the width of the operating face of the grinding circle and the machined surface.

The detail hookup along the length with the least value of the grinding surface width is considered to be preferable in choosing the grinding schema. So, having the analytical expression of the smallest and largest shape inaccuracy, it is possible to define the density of the flat joint surface of the face-grind circle.

The profile diagram analysis (Figure 6) removed from machined surface shows that, by flat grinding with the 
abrasive circle surface in accordance with the theoretical prerequisites made above, the nonstationarity of the roughness and the greatest value of the waviness and shape inaccuracy are generally observed along the length of the processing in input and output areas of the grinding circle in contact with the machined surface and offset area. The characteristic value of the micro- and macromisalignment in the input area is happened to be bigger than that in the output area. The average shape inaccuracy value in the input area comprised $22 \mathrm{mkm}$ and $18 \mathrm{mkm}$ in the output area and $42 \mathrm{mkm}$ in the offset area.

The control of the table in the length of the processing as well as the increase of the accuracy creates favorable conditions to increase the process efficiency [13-17]. Meanwhile, the increase in process efficiency is observed as the result of

(i) the increase of alignment in each pass;

(ii) changes in speed of the machine table in the length of the pass;

(iii) the performance of operations with high cutting modes which assists in expedited setting of the necessary tension in the system and allows a decreasing number of passes.

The results of the calculations demonstrate that the main process time decreases approximately by 1.8 times in the programmed change of the machine table speed.

The findings are in fact capable of achieving high economic efficiency of production. The results of the calculations and measurements showed average savings of 18 percent of the operating costs.

Works of the same nature on the subject could not be found even after an extensive literature review of similar orientation. For similar works describing the process readers are referred to James et al., 2009 and Fan et al. 2009. However, they only form a general theoretical base.

The economic efficiency is clear from the optimal setting machining parameters. Basic facts are reduced energy consumption, greater precision of machining, and less wear of the grinding wheel. Verification of the effectiveness is demonstrated by practical measurements.

\section{Conclusion}

(1) The given formula analysis shows that, by flat grinding with abrasive circle surface, the amount of the practically operating abrasive grains in the contact area depends on the size and the feature of the grinding wheel, as well as on the cutting mode elements.

(2) The most significant effects on the amount of active grains in the contact area were the width of surface circle of the cup grinding wheel; the ratio of the table speed to the circle speed, that is, the dimension of the so-called longitudinal feed to the circle revolution; the ratio of the circle diameter to the width of the processed detail; and so forth.

(3) The quantity is active the working abrasive grains in a contact zone at face grinding much more it is more, than at other types of grinding that is explained by bigger sizethe areas of contact at face grinding.

(4) Having analytical expression of regularity of change of quantity actually working abrasive grains within the technological primitives of an entrance, the established grinding and exit, from preservation conditions stability of amount of active abrasive grains, by means of change speeds of longitudinal giving, it is possible to provide the uniform abrasive impact on the processed surface.

(5) From the analytical statements issues it is possible to determine the economic calculations of the cost savings based on specific operating parameters. Moreover, it is necessary to derive the underlying mathematical expression. This task is the subject of the further work.

\section{Variables and Symbols}

$b$ : Width of a working surface of a grinding wheel, mm

$D_{c}$ : Pitch diameter of working face grinding

$S_{l}$ : Longitudinal feed per revolution of the disk, mm/v

$D_{c j}$ : Pitch diameter of $j$ conventional line

$V_{d}$ : Speed of the longitudinal feed of the machine table $\mathrm{m} / \mathrm{min}$

$V_{k}$ : Rational rate of the grinding disk, v/s

$D_{k p}$ : Outer diameter of the working face grinding

$F_{j}$ : Area of $j$ conventional disk of operating face grinding

$\beta$ : $\quad$ Correction for symmetric array of the curve, radius disposal of grains top on the working wheel layer

$\gamma$ : Half of the probable angle value of cutting grains apex;

$\omega:$ Specific capacity; mms.

$k$ : Concentration factor; \%

$\alpha$ : Coefficient of granular

$\bar{X}_{j}$ : Average value of grains, size of microgrit.

\section{Conflict of Interests}

The authors declare that there is no conflict of interests regarding the publication of this paper.

\section{Acknowledgments}

This project is cofinanced by funds from the European Union, ITMS Project Code 26220220125. IMAP; European Regional Development Fund, The European Union; Agency of the Ministry of Education, Science, Research and Sport of the Slovak Republic for the EU Structural Funds; and Operational Programme Research and Development support research activities in Slovakia. 


\section{References}

[1] H. A. Husseinov, Program Control Like Machine Processing, Chashioqli, Baku, Azerbaijan, 2000.

[2] B. Kilundu, X. Chiementin, and P. Dehombreux, "Singular spectrum analysis for bearing defect detection," Journal of Vibration and Acoustics, vol. 133, no. 5, Article ID 051007, 2011.

[3] E. Ragan, P. Baron, and J. Dobransky, "Sucking machinery of transport for dosing granulations of plastics at injection molding," in Proceedings of the International Conference on Manufacturing Science and Technology, Singapore, September 2011.

[4] A. Das and J. P. Misra, "Experimental investigation on surface modification of aluminum by electric discharge coating process using TiC/Cu green compact tool-electrode," Machining Science and Technology, vol. 16, no. 4, pp. 601-623, 2012.

[5] BSIa (British Standards Institute), “BS 2007:1983 Specification for circular gear shaving cutters, 1 to 8 metric module, accuracy requirements".

[6] BSIb, "Specification for rotary form relieved gear cuttersdiametral pitch," British Standards BS 2518 Pt-1, British Standards Institute, 1983.

[7] BSIc (British Standards Institute) BS 2518 Pt-2:1983 specification for rotary relieved gear cutters-metric module.

[8] S. Baragetti and F. Tordini, "Experimental and numerical study of shot peened thin hard-coated components," Mechanics Based Design of Structures and Machines, vol. 39, no. 4, pp. 441-460, 2011.

[9] A. N. Reznikov and M. Machinery, Eds., Abrasive and Uncut Diamond Processing of Materials. Reference Book, Loading Automation of Round Grinding Machine with N.P.C., 1977.

[10] E. N. Maslov, Theory of Grinding of Materials: M.: Mechanical Engineering, 1974.

[11] S. A. Bagirov and H. Husseinov, "Abrasive wheel for processing the details," Patent Invention no. I 2012 0008, Baku, Azerbaijan, 2012.

[12] H. A. Huseynov and S. A. Bagirov, "The analysis of the joint characteristics, based on the processing methods of the contacting surfaces," International Journal of Advances in Enjineering \& Technology, vol. 7, no. 2, pp. 318-326, 2014.

[13] G. G. Ahmad and B. S. Abbas, "Main features of the mechanism of formation the surface grunding with the periphery of a straight disk," International Journal of Advances in Enjineering \& Technology, vol. 7, no. 1, pp. 66-74, 2014.

[14] S. Hloch and J. Valíček, "Topographical anomaly on surfaces created by abrasive waterjet," International Journal of Advanced Manufacturing Technology, vol. 59, no. 5-8, pp. 593-604, 2012.

[15] K. A. James, J. S. Hansen, and J. R. R. A. Martins, "Structural topology optimization for multiple load cases using a dynamic aggregation technique," Engineering Optimization, vol. 41, no. 12, pp. 1103-1118, 2009.

[16] S.-K. S. Fan and J.-M. Chang, "A parallel particle swarm optimization algorithm for multi-objective optimization problems," Engineering Optimization, vol. 41, no. 7, pp. 673-697, 2009.

[17] T. G. Ritto, R. H. Lopez, R. Sampaio, and J. E. Souza de Cursi, "Robust optimization of a flexible rotor-bearing system using the Campbell diagram," Engineering Optimization, vol. 43, no. 1, pp. 77-96, 2011. 

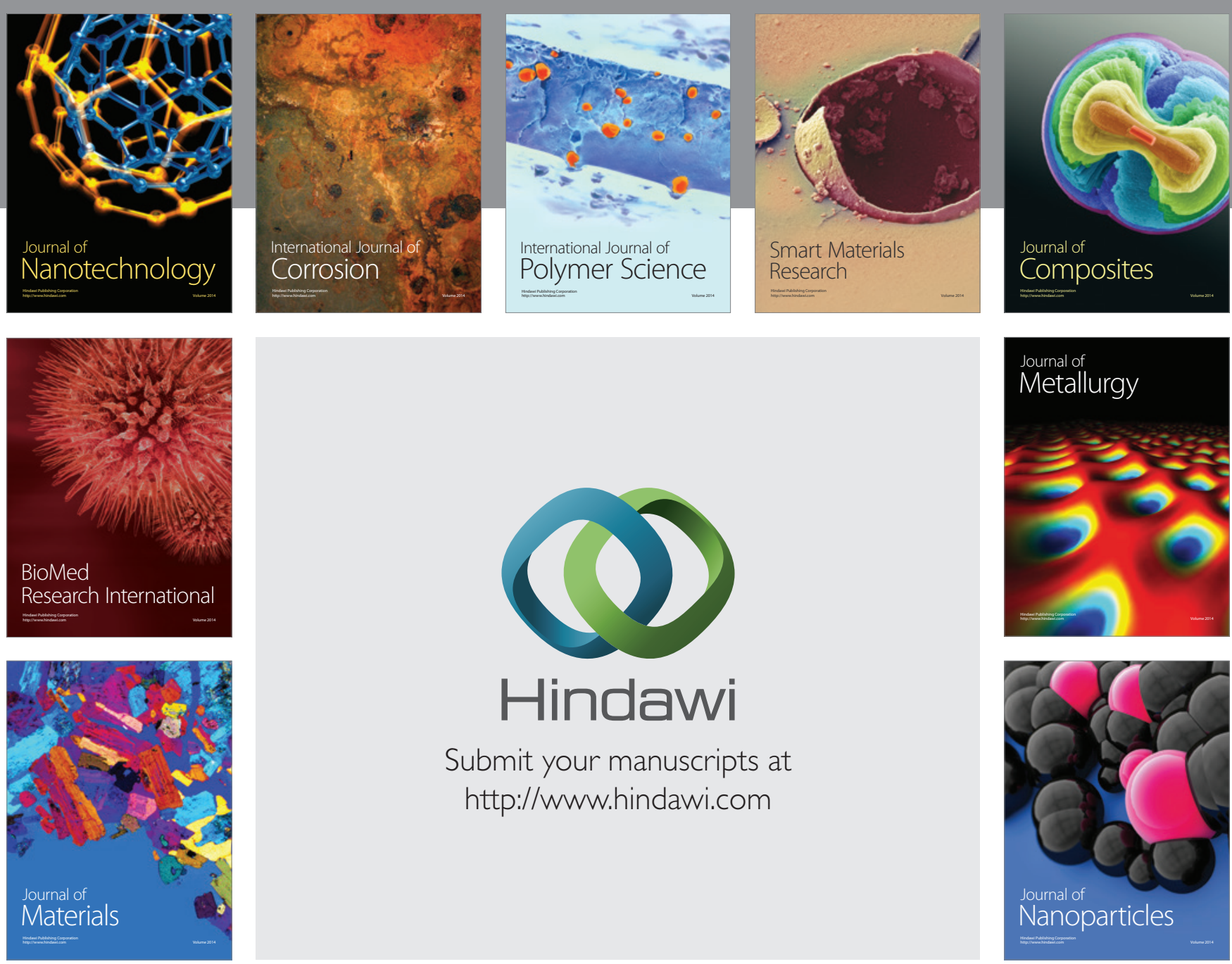

Submit your manuscripts at http://www.hindawi.com
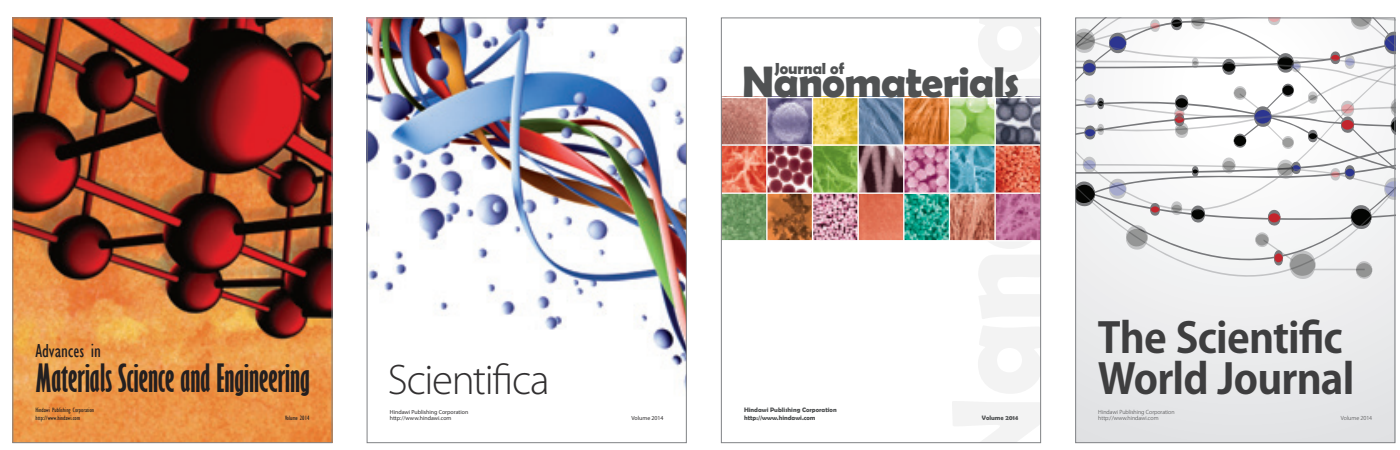

\section{The Scientific World Journal}
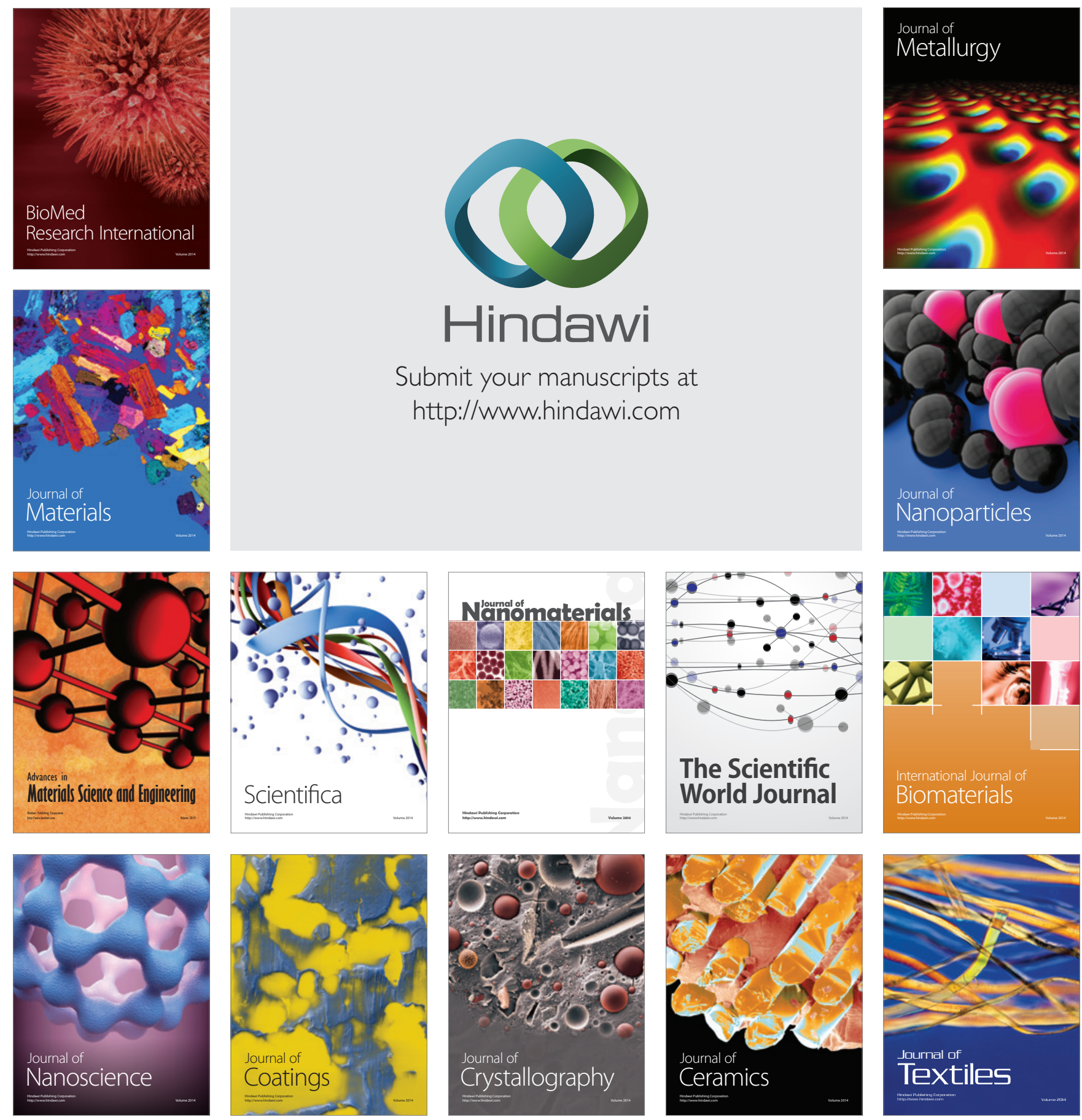